\title{
Informação adequada, confiável e oportuna em tempos de pandemia de COVID-19
}

\author{
Janine Giuberti Coutinho ${ }^{1}$ e Monica Padilla ${ }^{1}$
}

Como citar Coutinho JG, Padilla M. Informação adequada, confiável e oportuna em tempos de pandemia de COVID-19. Rev Panam Salud Publica. 2020;44:e118. https://doi.org/10.26633/RPSP.2020.118

RESUMO

Em tempos de pandemia da COVID-19, informação adequada, confiável e oportuna, torna-se mais ainda relevante. O escritório da Organização Pan-Americana da Saúde/Organização Mundial da Saúde no Brasil e parceiros, vêm desenvolvendo iniciativas para favorecer o acesso, compartilhamento e difusão das informações e conhecimentos produzidos, traduzidos, sistematizados sobre a COVID-19 como uma contribuição técnica para o enfrentamento da pandemia. Este artigo apresenta alguns desses recursos. É fundamental considerar como máxima relevância a produção e sínteses de conhecimento disponibilizado oportunamente para quem está na primeira linha de resposta à COVID-19. Isto como requisito básico para melhorar a capacidade de resposta. É importante também o fortalecimento e incremento nos investimentos para produção de ciência e evidência, e o reconhecimento e fortalecimento do incansável trabalho de um enorme contingente de cientistas e instituições que continuam imbuídas a responder à pandemia da COVID-19.

Palaras-chave Infecções por coronavírus; gestão do conhecimento; pandemias.

Em tempos de pandemia da COVID-19, informação adequada, confiável e oportuna, torna-se mais ainda relevante. Segundo o Diretor Geral da Organização Mundial da Saúde (OMS), Tedros Adhanom Ghebreyesus, "Não estamos apenas lutando contra uma epidemia; nós estamos lutando contra uma infodemia" [1,2]. A infodemia caracteriza-se como o excesso de informações, algumas precisas e outras não [3]. Informação adequada, confiável, oportuna e baseada em evidências é a melhor resposta que se pode dar em um momento de pandemia no qual ainda não temos uma vacina. A produção, disseminação de informação adequada e confiável perpassa as funções básicas da Organização Pan-Americana da Saúde (OPAS), a referência regional nas Américas para a OMS. A Organização aporta cooperação aos países, por meio da transferência de tecnologia e da difusão de informação e conhecimento em saúde pública $[4,5]$.

A renovação e a aplicação do conhecimento de maneira sistemática, explícita e deliberada com o objetivo de aumentar a possibilidade e capacidade de uma Organização atingir seus resultados pode ser entendida como Gestão do Conhecimento [6].

\footnotetext{
1 Organização Pan-Americana da Saúde, Brasília (DF), Brasil. $\square$ Janine Giuberti Coutinho, giubertjan@paho.org
}

No escritório da OPAS/OMS no Brasil, a Gestão do Conhecimento tem sido um motor para a promoção da cooperação técnica com o Ministério da Saúde e outros diversos parceiros em outros níveis federativos, com vistas a favorecer a implementação de intervenções, baseadas em conhecimentos científicos, as quais melhorem a saúde e o bem-estar das populações.

Em geral, diante da sua natureza investigativa, a produção de dados, informações, conhecimentos e sua disseminação leva tempo. No entanto, dada a urgência de resposta da OPAS à pandemia da COVID-19, impõe-se o aumento na velocidade de produção de conhecimento confiável e científico para ser colocado à disposição aos países para tomada de decisão. A disponibilização ágil e oportuna às informações técnicas e científicas reforça o papel estratégico da Organização. O objetivo é facilitar ao gestor, ao profissional de saúde e à população em geral informação para o seu uso em momento adequado. Neste contexto, o Escritório da OPAS/OMS no Brasil vem desenvolvendo um conjunto de atividades para a produção, tradução, sistematização e compartilhamento de conhecimento oportuno, adequado e estratégico como uma contribuição técnica para o enfrentamento da pandemia da COVID-19. Este é um trabalho inteprogramático, articulado com os escritórios regional (OPAS), a OMS e os parceiros estratégicos como o Centro 
Latino-Americano e do Caribe de Informação em Ciências de Saúde (Bireme) e o Campus Virtual de Saúde Pública.

Com o objetivo de favorecer o acesso, compartilhamento e difusão das informações e conhecimentos foi idealizado um espaço virtual para os profissionais de saúde (https://opascovid.campusvirtualsp.org/taxonomy/term/36) [7] no site da OPAS/OMS Brasil [8]. Neste espaço estão disponíveis:

- Cursos da OMS, elaborados pela Open WHO [9], traduzidos pela OPAS/OMS Brasil e hospedados no Campus Virtual de Saúde Pública. São cursos à distância, sem tutoria. Estes cursos também estão disponíveis nas plataformas do UNASUS e AVASUS, e em 19 Escolas Estaduais de Saúde Pública como estratégia chave para reforçar as capacidades dos professionais da saúde para enfrentar a COVID-19 e para se proteger com o adequado uso dos Equipamentos de Proteção Individual (tabela 1).

- Documentos, Orientações provisórias, Protocolos, Resumos científicos. Publicações, em diferentes formatos, produzidos pela OPAS e OMS. Conhecimento em temas como vigilância, laboratório, assistência à saúde, incluindo atendimento clínico, medidas não farmacológicas, comunicação de risco, e outros (tabela 1). Todos estes documentos oficiais, atualizados sistematicamente e referentes à resposta a COVID-19 pelas equipes de saúde no contexto da emergência.

- Experiências nacionais e internacionais (tabela 1) sobre o enfrentamento da COVID-19. No caso das experiências nacionais, estão apresentadas as respostas dos estados e municípios à COVID-19 orientados pela Atenção Primária em Saúde (APS). São experiências locais em oito linhas temáticas: (1) Organização das Unidades Básicas de Saúde na APS; (2) Organização dos serviços de APS para garantir o acesso e o cuidado continuado das pessoas que já são acompanhadas pelas equipes; (3) Saúde e segurança dos profissionais da APS; (4) Uso de tecnologia de informação e teleatendimento; (5) Vigilância em saúde a partir da APS; (6) Potencialização das ações intersetoriais; (7) Educação permanente das equipes; (8) Instituição de comitês técnicos e/ ou de gestores para o enfrentamento da pandemia. A OPAS, em parceria com o Ministério da Saúde, vem divulgando

Tabela 1. Conteúdos disponíveis no espaço virtual “Orientações Técnicas da Organização Pan-Americana da Saúde/Organização Mundial da Saúde para Profissionais da Saúde"

\begin{tabular}{|c|c|c|}
\hline \multirow{6}{*}{$\begin{array}{l}\text { Cursos da OMS traduzidos para } \\
\text { o português e disponíveis no } \\
\text { Campus Virtual de Saúde Pública }\end{array}$} & $\begin{array}{l}\text { Precauções básicas: higienização das mãos (COVID-19) } \\
\text { - } 2020\end{array}$ & $\begin{array}{l}\text { https://www.campusvirtualsp.org/pt-br/curso/ } \\
\text { covid-19-precaucoes-basicas-higienizacao-das-maos-2020 }\end{array}$ \\
\hline & $\begin{array}{l}\text { COVID-19: Como colocar e remover equipamentos de } \\
\text { proteção individual } 2020\end{array}$ & $\begin{array}{l}\text { https://www.campusvirtualsp.org/pt-br/curso/covid-19-como- } \\
\text { colocar-e-remover-equipamentos-de-protecao-individual-epis-2020 }\end{array}$ \\
\hline & $\begin{array}{l}\text { Doenças ocasionadas por vírus respiratórios emergentes, } \\
\text { incluind o o COVID-19 }\end{array}$ & $\begin{array}{l}\text { https://www.campusvirtualsp.org/pt-br/curso/doencas-ocasionadas- } \\
\text { por-virus-respiratorios-emergentes-incluindo-covid-19 }\end{array}$ \\
\hline & $\begin{array}{l}\text { Prevenção e controle de infecções causadas pelo novo } \\
\text { coronavírus (COVID-19) }\end{array}$ & $\begin{array}{l}\text { https://www.campusvirtualsp.org/pt-br/curso/prevencao-e-controle- } \\
\text { de-infeccoes-pci-causadas-pelo-novo-coronavirus-covid-19 }\end{array}$ \\
\hline & $\begin{array}{l}\text { Cuidados Clínicos na Síndrome Respiratória Aguda } \\
\text { Grave - } 2020 \text { (COVID-19) }\end{array}$ & $\begin{array}{l}\text { https://www.campusvirtualsp.org/pt-br/curso/cuidados-clinicos-na- } \\
\text { sindrome-respiratoria-aguda-grave-2020-covid-19 }\end{array}$ \\
\hline & $\begin{array}{l}\text { Projeto para Unidade de Tratamento de Síndrome Respiratória } \\
\text { Aguda Grave } 2020 \text { (COVID-19) }\end{array}$ & $\begin{array}{l}\text { https://www.campusvirtualsp.org/pt-br/curso/projeto-para-unidade- } \\
\text { de-tratamento-de-sindrome-respiratoria-aguda-grave-srag-2020 }\end{array}$ \\
\hline \multirow{16}{*}{$\begin{array}{l}\text { Documentos e publicações da } \\
\text { OPAS e OMS traduzidos para } 0 \\
\text { português }\end{array}$} & Aleitamento Materno e COVID-19 & https://opascovid.campusvirtualsp.org/taxonomy/term/74 \\
\hline & Prevenção e Controle de Infecções/WASH & https://opascovid.campusvirtualsp.org/taxonomy/term/47 \\
\hline & Guias Clínicas & https://opascovid.campusvirtualsp.org/taxonomy/term/43 \\
\hline & Guias Laboratoriais & https://opascovid.campusvirtualsp.org/taxonomy/term/44 \\
\hline & Vacinas e COVID-19 & https://opascovid.campusvirtualsp.org/taxonomy/term/60 \\
\hline & Outras patologias & https://opascovid.campusvirtualsp.org/taxonomy/term/59 \\
\hline & Direitos Humanos, Gênero e o Enfrentamento da COVID-19 & https://opascovid.campusvirtualsp.org/taxonomy/term/56 \\
\hline & Organização e Fortalecimento dos Serviços de Saúde & https://opascovid.campusvirtualsp.org/taxonomy/term/42 \\
\hline & Medicamentos e Insumos para a Saúde & https://opascovid.campusvirtualsp.org/taxonomy/term/55 \\
\hline & Trabalhadores de Saúde & https://opascovid.campusvirtualsp.org/taxonomy/term/50 \\
\hline & Saúde Mental e Apoio Psicossocial & https://opascovid.campusvirtualsp.org/taxonomy/term/51 \\
\hline & $\begin{array}{l}\text { Medidas Não Farmacológicas para a Redução da } \\
\text { Disseminação do Vírus }\end{array}$ & https://opascovid.campusvirtualsp.org/taxonomy/term/48 \\
\hline & Pesquisas e Protocolos de Investigação & https://opascovid.campusvirtualsp.org/taxonomy/term/52 \\
\hline & $\begin{array}{l}\text { Preparação e Prontidão e Ações de Respostas Crítica ao } \\
\text { COVID-19 }\end{array}$ & https://opascovid.campusvirtualsp.org/taxonomy/term/41 \\
\hline & Sensibilização e Orientação da População & https://opascovid.campusvirtualsp.org/taxonomy/term/49 \\
\hline & Saúde Indígena & https://opascovid.campusvirtualsp.org/taxonomy/term/62 \\
\hline \multirow{2}{*}{$\begin{array}{l}\text { Experiências de enfrentamento à } \\
\text { COVID-19 }\end{array}$} & Nacionais & https://opascovid.campusvirtualsp.org/taxonomy/term/65 \\
\hline & Internacionais & https://opascovid.campusvirtualsp.org/taxonomy/term/64 \\
\hline
\end{tabular}

OMS, Organização Mundial da Saúde; OPAS, Organização Pan-Americana da Saúde 
imediatamente as práticas inovadoras tanto no site https:/ / opascovid.campusvirtualsp.org quanto no www.apsredes. org. É fundamental dar ampla visibilidade às repostas que os municípios, estados estão dando à COVID-19, em tempo ágil e oportuno. Em momento posterior será realizado um prêmio APS Forte no SUS com a seleção e o reconhecimento das experiências bem-sucedidas.

Os documentos são produzidos e traduzidos para o português em função da demanda do Ministério da Saúde e outros parceiros como as Secretarias Estaduais e Municipais de Saúde. Para além do espaço virtual os conteúdos estão também disponíveis na Vitrine do Conhecimento da COVID-19 produzida e atualizada pela Bireme, disponível em https://bvsalud.org/ vitrinas/post_vitrines/novo_coronavirus/. E, também em aplicativo produzido pela OMS e traduzido para o português como uma outra maneira de disseminação ágil da informação (http:/ / www.academy.who.int/).

Vivemos uma das piores situações sanitárias da história, com a perda de quase 100 mil vidas no Brasil e quase 700 mil no mundo todo, além de todas as situações de privação e desigualdades que foram aprofundadas dadas às condições sociais, trabalho, renda e alimentação das populações no mundo. Com toda esta situação, o pesar da perda de muitas e muitas vidas, é fundamental considerar como máxima relevância a produção e sínteses de conhecimento disponibilizado oportunamente para quem está na primeira linha de resposta à COVID-19 como requisito básico para melhorar a capacidade de resposta. É fundamental o fortalecimento e incremento nos investimentos para produção de ciência e evidência, bem como o reconhecimento do incansável trabalho de um enorme contingente de cientistas, instituições que continuam imbuídas a responder da melhor forma possível à pandemia da COVID-19. Além da resposta imediata e contínua à COVID-19 que vem sobrecarregando seus sistemas de saúde e economia nos países, estes deverão também avaliar suas respostas, identificar suas lições aprendidas com o objetivo de reorganização dos sistemas e serviços de saúde nos anos vindouros, além da continuidade às pesquisas em diversas áreas do conhecimento.

Contribuição dos autores. JGC contribuição substancial para a concepção e projeto, aquisição de dados, elaboração do artigo. MP concepção, revisão do conteúdo; ambos dois autores aprovação da versão final.

\section{Financiamento. Nenhumo.}

Agradecimentos. Dra. Socorro Gross; Dra Maria Almiron, Rachel de Souza e toda a equipe do Escritório da OPAS/OMS Brasil.

Conflito de interesses. Nada declarado pelos autores.

Declaração. As opiniões expressas no manuscrito são de responsabilidade exclusiva dos autores e não refletem necessariamente a opinião ou política da RPSP/PAJPH ou da Organização Pan-Americana da Saúde (OPAS).

\section{REFERÊNCIAS}

1. Zarocostas J. How to fight an infodemic. Lancet. 2020;395(10225):676. https://doi.org/10.1016/S0140-6736(20)30461-X

2. The Lancet Digital Health. Pandemic versus pandemonium: fighting on two fronts. Lancet Digital Health. 2020;2(6):e268. https:/ / doi.org/10.1016/S2589-7500(20)30113-8.

3. Organização Pan-Americana da Saúde. Entenda a infodemia e a desinformação na luta contra a COVID-19. Washington, DC: OPAS; 2020. [acesso em 12 agosto de 2020]. Disponível em: https://iris. paho.org/handle/10665.2/52054

4. Organização Pan-Americana da Saúde. Plano Estratégico da OPAS 2020-2025. [acesso em 12 agosto de 2020] Disponível em: https:// iris.paho.org $/$ handle $/ 10665.2 / 51599$ ?locale-attribute $=$ pt

5. Organização Pan-Americana da Saúde. Portfólio de cooperação técnica OPAS/OMS. Representação no Brasil. $2^{a}$ Edição. Brasília, D.F.: OPAS; 2018.

6. Organização Pan-Americana da Saúde. Estratégia de Gestão do Conhecimento. [acesso em 12 agosto de 2020] Disponível em: https://www.paho.org/es/documentos/estrategia-plan-accion-so bre-gestion-conocimiento-comunicaciones
7. Organização Pan-Americana da Saúde [página na internet]. Informações sobre a COVID-19 para profissionais de saúde [acesso em 12 agosto de 2020]. Disponível em: https://opascovid.campusvirtualsp.org

8. Organização Pan-Americana da Saúde [página na internet]. OPAS/ OMS Brasil [acesso em 12 agosto de 2020]. Disponível em: https:/ / www.paho.org/bra

9. World Health Organization. [página na internet]. Open WHO [acesso em 12 agosto de 2020]. Disponível em: https:/ /openwho.org/

10. Organização Pan-Americana da Saúde [página na internet]. Bireme [acesso em 12 agosto de 2020]. Disponível em: https://bvsalud. org/vitrinas/post_vitrines/novo_coronavirus/

Manuscrito recebido em 28 de julho de 2020. Aceito em versão revisada em 13 de agosto de 2020 


\section{Adequate, reliable and timely information in times of the COVID-19 pandemic}

ABSTRACT In times of the COVID-19 pandemic, adequate, reliable and timely information becomes even more relevant. The Pan American Health Organization/World Health Organization office in Brazil and key partners have developed initiatives to improve access, sharing and dissemination of the information and knowledge produced, translated and systematized about COVID-19 as a technical contribution to the fight against the pandemic. This article presents some of these resources. The production and synthesis of knowledge made available in a timely manner to professionals on the frontline of response to COVID-19 is essential and a basic requirement for improving response capacity. It is also important to strengthen and increase investment in the production of scientific evidence, and to recognize and build upon the tireless work of a large group of scientists and institutions that continue to respond to the COVID-19 pandemic.

Keywords Coronavirus infections; knowledge management; pandemics.

\section{Información adecuada, fiable y oportuna en tiempos de la pandemia por la COVID-19}

RESUMEN

Palabras clave
En tiempos de la pandemia por la COVID-19, la información adecuada, fiable y oportuna se vuelve aún más relevante. La oficina de la Organización Panamericana de la Salud/Organización Mundial de la Salud en el Brasil y sus contrapartes han desarrollado iniciativas para favorecer el acceso, el intercambio y la difusión de la información y los conocimientos producidos, traducidos y sistematizados sobre la COVID-19 como contribución técnica a la lucha contra la pandemia. Este artículo presenta algunos de estos recursos. Es fundamental considerar como de máxima relevancia la producción y síntesis de conocimientos puestos a disposición de manera oportuna para los profesionales que se encuentran en la primera línea de respuesta a la COVID-19; este es un requisito básico para mejorar la capacidad de respuesta. También es importante fortalecer y aumentar la inversión en la producción de evidencia científica, y reconocer y fortalecer la labor incansable de un enorme grupo de científicos e instituciones que siguen respondiendo a la pandemia por la COVID-19.

Infecciones por coronavirus; gestión del conocimiento; pandemias. 